\title{
Discursive construction of migrant otherness on Facebook: A distributional semantics approach
}

\author{
Victoria Yantseva*
}

\begin{abstract}
This work aims to study the social construction of migrant categories and immigration discourse on Swedish-speaking Facebook pages in the last decade. It combines the insights from computational linguistics and the distributional semantics approach with those from classical sociological theories to explore a corpus of more than $1 \mathrm{M}$ Facebook posts. This allows one to compare the meanings of labels denoting various categories of migrants and identify the interpretative repertoires used by Facebook users to discuss the immigration topic. The study concludes that, despite the expressions of tolerance and support for refugees and immigrants, the Facebook audience is nevertheless active in the objectification and discursive discrimination of those identified as belonging to either of those discursive categories. The study results are then related to the technological design of new media and the overall social and political climate surrounding the Swedish immigration agenda.
\end{abstract}

Keywords: word vectors, vector space models, word2vec, doc2vec, Facebook, social media, corpus linguistics, discourse analysis, migration

\section{Introduction}

Language is an essential part of our everyday interactions in society that helps us make sense of the things we see, hear and feel, and can be named a necessary component in constructing social reality. Human migration, for instance, has been named as one of such social constructs that are collectively created through language and discourse. Media have been argued to be one of the most potent agents with respect to their opportunities to influence the representation of migrants in the public space and reproduce discrimination [1]. At the same time, language and communication have been named as essential means for discursive reproduction of racism [2, p.28]. Social media seem to have a particularly peculiar role in these processes, fueling these tendencies and giving voice to those usually absent from the mainstream public arena, for example, right-wing parties and nationalist movements [3].

In this article thus, I study Facebook as a medium where social reality takes form primarily through written text and where the idea of migration and images of migrants are constructed and made sense of by those using this medium. In particular, I combine the insights from computational linguistics and distributional semantics approach with

\footnotetext{
*Department of Social Studies, Centre for Data Intensive Sciences and Applications, Linnaeus University, Sweden Universitetsplatsen 1, Växjö, 35252, Sweden victoria.yantseva@lnu.se
} 
discourse theory in order to study the use of language for the construction of public discourse on immigration and corresponding immigrant and refugee categories on the Swedish-speaking Facebook pages and groups. My ambition is to understand to what extent this discourse is complicit with the representation of immigrants and refugees as belonging to the category of the Other. Thus, I ask two questions:

(1) Which interpretative repertoires are used in the corpus to construct the immigration discourse?

(2) What are the differences in the construction of the "refugee" and "immigrant" categories in the Facebook discourse?

To answer these questions, I use a mixed-methods approach. The computational part of the study is represented by the distributional semantics approach, in particular, by word embeddings (word2vec and doc2vec models) to map the location of words and documents from the corpus in a multidimensional semantic space. The core of the distributional semantics approach can be best expressed by a famous quote: "you shall know a word by the company it keeps" [4], or an idea that one can conceive of a word's meaning by studying the context in which it is used. Although word embeddings have been an increasingly popular research tool, their use in social sciences and social media studies has mostly been limited (for some exceptions, see, among others, [5, 6]). Moreover, the use of word embeddings for discourse analysis seems also to be a promising research area. However, this opportunity has not been efficiently addressed so far in the discourse studies (see [7, 8] as exceptions).

This study's ambition is to contribute to the existing research in several ways. One of them is to demonstrate a possibility for a successful combination of computational and interpretative approaches in discourse studies and social media studies in general. In particular, the paper demonstrates that vector-space models can help researchers combine high-level quantitative analysis of the whole corpus with a more qualitative and close reading of individual documents. Moreover, this study seeks to contribute to the existing research on migrant categorisations by using a quantitative approach that makes it possible to validate the results of the previous studies that were often conducted with the help of qualitative methods. Further, while a large part of discourse analytic studies have focused on the construction of differences between "native" and "non-native" populations, this work aims to provide new insights into the differences in the construction of specific migrant categories. Finally, it is especially interesting to focus on Facebook, a less studied social medium in comparison with, for instance, Twitter, because of the previously existing restrictions on using its data.

The study uses a corpus of messages posted in 2012-2020 on the public Swedishspeaking Facebook pages and groups $(\mathrm{N}=\sim 1,07 \mathrm{M})$. Although Sweden can be described as a relatively small European country in comparison with some of its' European counterparts, it represents an interesting case of a seemingly colour-blind society [9] that has been striving to ensure social inclusion of its inhabitants irrespective of their ethnic background [10]. It is especially interesting to study if, and to what extent, migration and migrants are constructed with reference to the categories of whiteness and otherness in such a colour-blind society. Since Facebook is one of the most popular social networks in Europe, I use this platform as an example of participatory media that plays 
an increasingly important role in the definition of public agenda worldwide.

\section{Related work}

Mainstream and social media, quite evidently, can be named as one of the leading entities participating in the social construction and reproduction of migrant identities [1. In the European context, for instance, it has been pointed out that deserving refugees and undeserving migrants were presented as two contradictory positions during the European refugee crisis [11. While the latter ones were constructed as a threat to the receiving societies that needed to be expelled, the former group has been presented as requiring protection [12. It has been argued that "Persons seeking refuge in Europe must sustain an identity of 'non-threatening victim' if they are to gain recognition in a securitised culture of (mis)trust" [13. On the contrary, immigrant identities, it has been suggested, are constructed in such a way that a boundary between the Europeans and "criminal outsiders" is created [14]. At the same time, the presence of immigrants was directly attributed to the terrorist threat to the host societies [15]. On the other hand, some of the previous studies have also provided somewhat contradictory evidence that the refugee category was evaluated more negatively than the immigrant one [16].

Nevertheless, such a "categorical fetishism" has been argued to be a distinctive trait of the current European securitised immigration regime and discourse [17, 18. Moreover, the use of the two categories has been found to be temporal and dependent on the social and political climate in the host societies [12]. Obviously, such an obsession with migrant labelling is active in the reproduction of inequalities and exclusion and cannot reflect the whole complexity of migratory experiences that not always easily fall within one of the two categories [18].

Swedish social research, however, has primarily addressed the issue of migrant categorisations focusing on the construction of differences between the Swedish and "nonSwedish" population with the help of references to the concepts of otherness and whiteness [9, 19]. Another relevant insight about this general distinction between Swedes and migrants is that migrantness has an entirely different meaning in the public discourse. This position is not based on the experience of migration but rather on a degree of non-conformity with the existing norms [20, p. 50]. Further, an analysis of discourse on Facebook has shown that there exist three "cybertypes": immigrants, Swedes and politically correct elites [21. Nevertheless, the existing studies have generally given less attention to the systematic exploration of this distinction between various labels and categories of migrants. The existing research on this topic is limited by the evidence on the diverging attitudes of the Swedish population towards immigrants and refugees that rely on ethnic origin, economic situation in the home country and home region as the main determinants of these attitudes [17].

The European refugee crisis of 2015-2016 has even further problematised this distinction between the native white population and the Other. At the core of this distinction, as argued by the researchers, is the notion of whiteness that seems to play the key role in the construction of the European identity and that comes into being with the help of a juxtaposition against the non-white population [22]. Such a practice of migrant othering has been described as a form of cultural racism and a form of symbolic violence since it primarily includes overt forms of discrimination based on social or cultural difference 
[23. p. 1-3]. The events of 2015-2016, accordingly, have brought up to the surface the crisis of this European identity and an "unresolved racial crisis" in the broader sense 24]. Yet, as the scholars have correctly suggested, the nomination of any events as a crisis in the first place needs to be problematised since it presumes that the crisis is preceded by the period of stability or normality [25]. This argumentation is directly applicable to the Swedish case. In particular, a relevant argument is that the ground of this crisis in Sweden can be found in a breakdown of somewhat contradictory images of "old Sweden" as "people's home" and a strong welfare state, on the one hand, and "good Sweden" as a supporter of human rights, anti-racism and anti-colonialism, on the other hand [26, 9].

It seems that social media play an increasingly important role in constructing biased perceptions of migrants and the reproduction of inequalities. An extensive body of work has demonstrated, for instance, that exclusionary rhetoric has been quite common on the Twitter platform during the refugee crisis and afterwards [14, 27]. Even more so, the research has documented the widespread use of social media, including Facebook, by populist movements [28, 29]. In the Nordic context, the specifics of populist and right-wing rhetoric in social media is that it essentially represents migration as a threat to the traditionally strong welfare system [30]. One more work of Swedish researchers has shown that the Facebook audience employed a range of specific perspectivising and persuasive strategies in order to contribute to a racist discourse [21].

\section{Theoretical framework}

This paper is inspired by and builds its theoretical framework upon the discourse theory, first and foremost the writings of Jonathan Potter and Margaret Wetherell [31, 32, 33], who refer to discourse as a term "...to cover all forms of spoken interaction, formal and informal, and written texts of all kinds" [31, p. 7]. Just as most discourse theorists, they question the role of language as a tool for mere representation of the social world and acknowledge the role of language and discourse in the construction of social reality. What makes their approach stand out in comparison with some of the discourse theories is that they also emphasise that there exists duality in relation to the discourse - on the one hand, agents are "...strategically using discourse" [32, p. 93], and, on the other hand, discursive forms are "...playing themselves out through the actions of individuals" [32, p. 93].

Thus, the researchers recognise the power of individuals to interpret events and social phenomena and choose from a variety of interpretative repertoires, or "broadly discernible clusters of terms, descriptions and figures of speech assembled around metaphors and vivid images" [32, p. 90]. The concept of interpretative repertoires comes in extremely handy in the corpus-based studies of discourse since the former help to uncover the content of discourse and a variety of existing and often competitive interpretations of the same phenomenon [32, p. 90] that can be subject to high variability and contextuality. The first discourse analytic task in this paper, therefore, is to uncover this interpretative repertoire that social media users choose from when discussing immigration on social media since the idea of clusters of terms that denote a particular repertoire is fully coherent with the modern document clustering techniques used in computational text analysis. 
The idea of meaning also has a very special role not only in discourse studies but also in relation to the vector-space representation of words. The distinction between the form and meaning is best reflected with the help of a famous notion of a sign that consists of a signified and a signifier [31, p. 25], which also points at the fact that "There is nothing that determines the nature of the signifier or the nature of signified; there is no intrinsic relationship between them" [31, p. 25]. In relation to the immigration agenda, it is interesting to compare how the meanings (signified) of various linguistic forms (signifiers), such as immigrant, refugee or undocumented migrant, are being constructed through the discourse. While, possibly, one can assume that there are some "dictionary" meanings of the words immigrant and refugee, the idea of signs and the statement about the contextual and constructive role of discourse allow suggesting that the meanings of the labels immigrant and refugee are not exhausted by their dictionary definitions.

With this regard, Potter and Wetherell discuss the notion of social categories whose members are presumed to possess certain qualities just because of the corresponding category membership [31, p. 117]. However, in contrast to the tradition of categorisation research in psychology, Wetherell and Potter point out that people's decisions to assign others to particular categories are highly variating and context-dependent - in other words, the same person can assign another person to different categories depending on the circumstances [31, p. 136]. On top of this, the categories themselves are actively reconstructed through the discourse [31, 136]. Therefore, the second analytic task is to study the construction of various categories of migrants through the discourse and to identify the actual meanings behind the labels of immigrants and refugees.

While classical discourse analysis is usually performed qualitatively by, for instance, reading a collection of texts, the age of big data allows performing the same task in an automated way with the help of natural language processing methods. Word2vec represents one of more sophisticated tools for computer-mediated discourse analysis in comparison with some classical methods such as the study of collocations or word keyness [34, p. 164]. The idea that word embeddings can be used in the study of discourse can be easily demonstrated with the following passage: "The choice, frequency and distribution of words can indicate what a segment of discourse is about (topicality), as well as communicators' attitudes and affective states" [35]. The choice, frequency and distribution of words are precisely what the distributional semantics approach is concerned with.

\section{Methods}

The use of computational methods for the analysis of language and textual data allows performing a distant reading of a large number of texts (a corpus) that constitute discourse (for a discussion on close and distant reading, see, for instance, [36]). Such a quantitative analysis of data has several strengths: it allows finding patterns in the data and testing their statistical significance, reduces researcher bias and helps avoid so-called cherry-picking, as well as makes it possible to work with a large amount of data [25, p. 156]. Such a quantitative analysis of text data can be further supplemented with a close (qualitative) reading of individual texts from the corpus [37]. The strength of the doc2vec approach, then, is that it allows combining distant and close reading of a 
corpus or an abstract overview of the corpus and a more specific focus on the qualitative aspects of the discourse. In this way, this study uses a mixed-methods approach that employs both distant and close reading of the corpus.

So how can the use of word2vec / doc2vec models be relevant to the study of the immigration discourse on social media? As mentioned above, discourse is associated with language use - and, therefore, the ambition of this study is to identify how language is used in the Facebook corpus to construct the various migrant categories, such as immigrant versus refugee, by studying the context in which these labels appear. The hypothesis is that words located near each other in a document also have similar meanings [38], or the similar idea is that "you shall know a word by the company it keeps" 4. Therefore, by studying how context words relate to the target labels, one can single out the meanings of these labels. We can then quantify the meanings of different words constituting the documents or documents constituting the corpus by assigning them with numeric vectors of $100 / 300$ dimensions where each dimension would represent some aspect or a sense of the word or document. The closer the two documents or words are in this semantic space, the more similar their meanings are to each other.

The data for this paper were collected in October 2020 - March 2021 via Facebook's platform CrowdTangle [39] that enables access to the Facebook posts published on the public pages and groups with more than 50 thousand members (followers) each. A total of $1.07 \mathrm{M}$ unique messages in Swedish dated from the 1st of January, 2012 to the 31st of December, 2020, were collected for the analysis. The search on CrowdTangle was performed with the help of keywords (e. g., "immigrants", "refugees", "immigration", "integration", "refugee policy", etc.). It is worth emphasising that this study is by no means representative of the Swedish Facebook discourse in general since collected data includes only data from public pages and groups, which leaves out posts published by users on their personal pages. Another constraint related to document sampling is that only documents in Swedish were used to build the model.

Raw text data were pre-processed: all URLs, e-mails, hashtags, mentions, numbers, stop words (built-in and custom) and punctuation marks were removed. All duplicated messages, as well as those containing less than five terms, were removed from the corpus. Messages in languages other than Swedish were identified automatically with the help of an automatic language detection tool (Python's fasttext library [40]) and also removed from the dataset 1 After the pre-processing, the resulting corpus included 983 thousand documents and $41.5 \mathrm{M}$ terms.

In this study, I used a doc2vec model, an extension of a famous word2vec model (aka word embeddings) that was first described in a seminal paper by Mikolov et al. [41. In a nutshell, word2vec learns a multidimensional vector-space representation of each word in a corpus by studying its closest neighbours in each document. In this multidimensional space, each dimension represents some sense of a word (for illustrative purposes, these can be colour, shape, size, etc.). Word2vec calculates each word's "score" on each of these dimensions and represents it in the form of a numeric vector. Semantically similar words (for example, "car" and "vehicle"), thus, tend to be located close to each other. Paragraph2vec model (or doc2vec, as it is also called), then, not only calculates such

\footnotetext{
${ }^{1}$ The code to reproduce the analysis is available at https://github.com/victoria-yantseva/ facebook-discourse
} 
vectors for individual words but also for the whole paragraphs or documents in a corpus 42. Therefore, I used an implementation of the doc2vec model (Distributed Bag of Words, DBOW) in Python's Gensim library [43] that learns word and document vectors jointly. The final model used for the analysis included 220 thousand unique terms.

Model hyperparameters were chosen with the help of QVEC-CCA, a method for word2vec evaluation proposed by Tsvetkov et al. [44. In short, QVEC-CCA compares learned word2vec vectors against a set of linguistic vectors compiled manually. Thus, following the work of Fallgren et al. [45], learned word embeddings were compared against the vectors built on supersenses from the Swedish word association lexicon SALDO [46]. QVEC-CCA was used to evaluate a range of models with the following parameters: window size $(8,10,12)$, epochs $(20,30,40)$ and dimensions $(100,200,300)$. The final model used in this work was trained with the following hyperparameters: distributed bag of words (DBOW), window size of 10, 300 dimensions, 40 epochs and a minimum word count of 5 . This model achieved a QVEC-CCA score of 0.313 , which is not far from the scoring of 0.357 for the general-purpose models that were trained by Fallgren et al. on a five times larger corpus [45].

It needs to be mentioned that much of current research on prediction-based vectorspace models has been performed on very large corpora of several hundreds of millions of words. However, it seems that a certain threshold to use word2vec is a corpus with roughly $1 \mathrm{M}$ tokens [47]. Some of the studies report, however, that word2vec can be effectively applied even for small-domain specific corpora of as little as 500 thousand words and that corpus specificity is a more critical parameter than corpus size [48. Hyperparameter setting, rather than corpus size, was found to have a determining value for the quality of learned word vectors [49]. Therefore, the used corpus size of more than $41 \mathrm{M}$ tokens seems to be appropriate since the goal is to create not a general purpose but rather a narrow task-specific model.

To extract the document clusters that would later be used to distinguish the primary interpretative repertoires, I applied a K-means clustering algorithm to divide semantically similar documents into groups based on their proximity to each other. Initially, the elbow criterion was used to find the optimal number of clusters. However, it did not allow finding the best partitioning due to a relatively gradual drop in the Sum of Squared Errors (SSE) values and the absence of an apparent elbow on the resulting plot. Therefore, the number of clusters was evaluated manually for $\mathrm{k}=6,8,10$ and 12 clusters, and a solution with $\mathrm{k}=8$ clusters was chosen based on the resulting clusters' interpretability. A specific limitation in relation to the choice of this clustering algorithm is based on the assumption that each document belongs only to one cluster (topic), which is not necessarily the case for all documents in the corpus.

To distinguish between various repertoires and to understand what the documents in a given cluster were primarily concerned with, TF-IDF weighting (term frequency inverse document frequency) was applied to the words populating documents in each of the document groups, which allowed distinguishing the most "important" words in each of the clusters. Top-30 tokens with the highest TF-IDF weights in each cluster were chosen as the keywords that would provide insight into the main repertoire of documents in the clusters, similar to how it is done in classic topic modelling. This was supplemented by the qualitative reading and analysis of the 50 documents located closest to each cluster centroids. In the qualitative part of the analysis, I did not follow 
any particular coding procedure. Instead, the task was to validate the correctness of themes identified with the help of keywords and then to find patterns and similarities between the documents belonging to the same cluster. The same procedure was applied to the documents chosen to contextualise the migrant labels.

To compare the discursive construction of the migrant categories, I performed several steps. I extracted the top-30 context words that had the highest cosine similarity scores with the two target labels (immigrants and refugees) [invandrare and flyktingar in Swedish]. In general, cosine similarity measure (ranging between 0 and 1) allows evaluating the document or term similarity by measuring the cosine of the angle between the vectors for the given word or document pairs. The use of cosine similarity measure, then, allowed identifying the closest neighbours of the target categories in the semantic space. To visualise this contextual and meaning-related component of the discursive categories in question, I built a semantic network where the two target labels represent seed labels and context words (those with cosine similarity higher than 0.55 and located on the paths of the maximum length of two) represent their closest neighbours.

Secondly, I compiled several lists of descriptive terms related to finances (costs and benefits), threat and solidarity issues that, as argued by the previous research, have been used especially frequently to construct the immigrant and refugee categories. These neutral word lists were supplemented with two more label lists (.e.g. "asylum seeker(s)", "refugee(-s)" versus "migrant(-s)", "immigrant(-s)". Following Garg et al. [5], I used Relative Norm Distance (RND) metric and its implementation in the wefe library in Python [50] to evaluate the relationship between these discursive categories and neutral word lists. The metric's leaning towards one of the two categories would signify that the category in question is more associated with the word list denoting the concept in question (threat, costs-benefits and solidarity).

Thirdly, using the cosine similarity measure, I identified top-50 documents located closest to each of the target categories in the learned multidimensional space, which provided an additional qualitative perspective of which utterances represented typical ways of talking about the two labels. In other words, these documents can be seen as the most typical or representative of conveying the meanings attached to the target labels.

\section{$5 \quad$ Results}

\subsection{Discursive construction of migrant categorisations}

As mentioned in the introductory section, the first goal of this work has been to compare how the two categories (immigrant versus refugee) are constructed. This goal was achieved by studying the semantic space produced by the doc2vec model and, specifically, by studying the location and closest neighbours of the target categories in this semantic space. A particularly interesting task has been to understand whether this contextualisation of the categories was performed in diverging ways and whether migrant categories were constructed with the help of the context words so that it sustained their representation as the Other.

The results suggest that the two categories are, in fact, constructed in somewhat different ways, as depicted in Figure 1. For instance, the labels denoting forced char- 


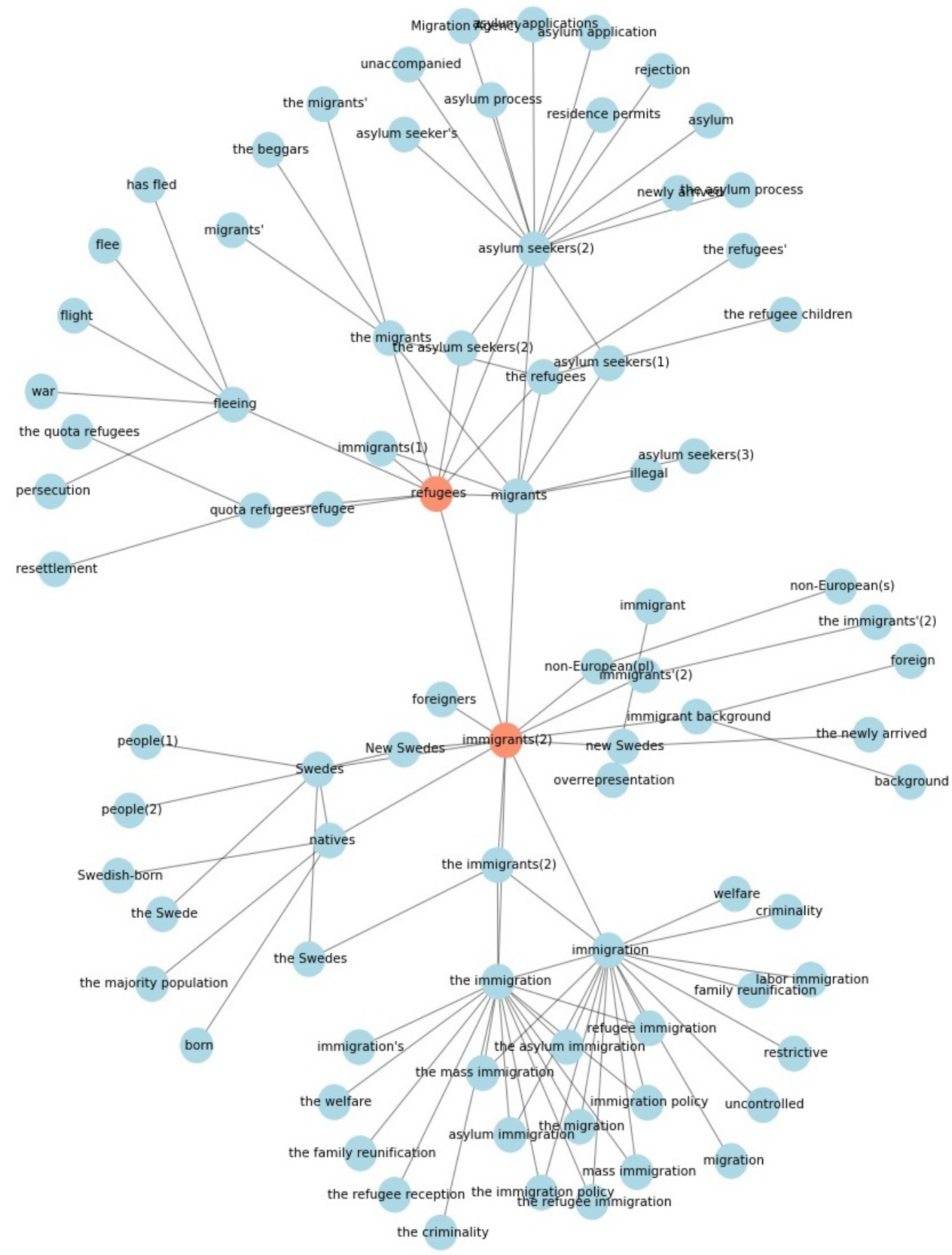

Figure 1: Semantic network of target and context words

Translation from Swedish. The closest neighbouring terms were chosen based on cosine similarity measure (see Methods section for more details). 
acter of migration ("asylum seeker(-s)" or "refugee(-s)") are located close to the words that are associated with the reasons behind emigration from the country of origin (e.g. "persecution", "resettlement", "residence permits", "war", "flight" etc.). The categories that roughly denote voluntary migration (i.e. "immigrants" and "immigration") are contextualised differently and are associated with the words that refer to the immigration policy and reception in the destination country ( e.g. "immigration policy", "restrictive", "uncontrolled", "criminality", and so on).

Table 1 presents the results of applying the Relative Norm Distance (RND) metric introduced in the Methods section to the three perspectives described as the most typical ones in the existing literature: these are costs and benefits, threat and solidarity perspectives. The initial assumption was that the immigrant category would be more associated with the perspectives that contextualise them as a threat to the receiving society (for instance, violating laws, entering the country illegally, and so on) or that would represent them as a group that can generate either economic profit or loss to the receiving society. It was also expected that the refugee category would be constructed as in need of help, protection and solidarity. These assumptions were, in fact, supported: the positive values of the RND metric for the "threat" and "cost and benefit" (0.08 and 0.06) perspectives denote a stronger bias towards or association with the voluntary type of migration. In contrast, negative values for the "solidarity" (-0.12) perspective denote a stronger association with the forced type of migration.

The results of qualitative reading of the messages allow providing a more nuanced picture of the differences between the ways in which immigrants and refugees are constructed. For instance, they indicate that Facebook authors tend to distinguish between the "right" and deserving type of immigrants (paying taxes, having employment and learning the Swedish language) that can be tolerated and let stay contrasted with the wrong and undeserving ones (undocumented, receiving social assistance, struggling with the language or committing crimes). The strategy of distinction between deserving and undeserving immigrants seems, in this case, to act as justifying possibly immigrant-hostile or prejudiced attitudes (author's translation below and elsewhere in the text):

(1) Why not introduce a well-behaviour requirement for those who come to Sweden... when criminality means deportation... I think this would benefit all those immigrants who behave well and want to participate in society. Not to mention that we Swedes would treat well those immigrants who behave themselves..

Qualitative reading of the messages supports the finding that the immigrant category is more frequently associated with the cost and benefit and threat perspectives, and these two perspectives seem to form the ground for the above-mentioned decision on immigrant deservingness. In other words, the reception of immigrants is constructed as an issue that needs to be decided upon based on the expected net profit or loss (including possible threats) that this category can bring to the receiving society.

It seems that, generally, immigrants and the Swedish are represented as two contradictory positions that come into being precisely as a result of a reference to and the contradiction with the opposite category: Swedishness is presented as "inborn", "ordinary" and "normal", whereas migrantness is something that is not. Nevertheless, 


\begin{tabular}{|l|c|l|l|l|l|}
\hline \multicolumn{2}{|l|}{ Cost and benefit perspective } & \multicolumn{2}{|l|}{ Threat perspective } & \multicolumn{2}{l|}{ Solidarity perspective } \\
\hline work & -0.09 & violence & -0.03 & reception & -0.47 \\
\hline cost & -0.07 & rapes & -0.03 & help (vb) & -0.31 \\
\hline profit & -0.06 & fear & -0.02 & $\begin{array}{l}\text { in need of pro- } \\
\text { tection }\end{array}$ & -0.26 \\
\hline $\begin{array}{l}\text { Employment } \\
\text { agency }\end{array}$ & -0.04 & attack & 0.00 & fellow people & -0.24 \\
\hline jobs & -0.03 & scared & 0.00 & compassion & -0.21 \\
\hline money & -0.02 & risk & 0.01 & help (nn) & -0.17 \\
\hline workplaces & 0.01 & threat & 0.02 & support (vb1) & -0.16 \\
\hline industry & 0.03 & criminal & 0.03 & distressed & -0.16 \\
\hline taxpayers & 0.03 & expulsion & 0.03 & need & -0.16 \\
\hline means & 0.03 & threatening & 0.04 & exposed & -0.14 \\
\hline economy & 0.04 & criminality & 0.05 & support (nn) & -0.13 \\
\hline companies & 0.04 & rapists & 0.06 & in need of help & -0.12 \\
\hline profitable & 0.04 & destroy & 0.06 & affected & -0.12 \\
\hline allowance & 0.05 & crime & 0.07 & integrate & -0.12 \\
\hline welfare & 0.05 & youth gangs & 0.08 & support (vb2) & -0.11 \\
\hline service & 0.05 & criminality & 0.09 & protection & -0.10 \\
\hline pensioners & 0.06 & attack (nn) & 0.09 & welcome & -0.09 \\
\hline costly & 0.06 & police & 0.10 & inclusion & -0.07 \\
\hline unemployed & 0.07 & ISIS & 0.10 & solidarity & -0.07 \\
\hline finance & 0.08 & attack (vb) & 0.10 & sympathy (1) & -0.06 \\
\hline employment & 0.08 & dangerous & 0.10 & community & -0.06 \\
\hline unemployment & 0.09 & violent & 0.11 & include & -0.06 \\
\hline GDP & 0.11 & terrorists & 0.12 & integration & -0.06 \\
\hline workforce & 0.12 & criminals & 0.12 & common & -0.05 \\
\hline small businesses & 0.13 & penalty & 0.14 & participation & -0.03 \\
\hline social assistance & 0.13 & damage & 0.15 & establishment & 0.00 \\
\hline support (vb) & 0.14 & illegal & 0.16 & sympathy (2) & 0.00 \\
\hline pensions & 0.16 & petty criminals & 0.25 & multiculturalism & 0.07 \\
\hline loss & 0.17 & illegal & 0.27 & diversity & 0.09 \\
\hline low-wage jobs & 0.19 & & & & \\
\hline wages & 0.20 & & & & \\
\hline taxes & 0.22 & & $\mathbf{0 . 0 8}$ & & \\
\hline Total scores: & $\mathbf{0 . 0 6}$ & & & & $\mathbf{- 0 . 1 2}$ \\
\hline & & & & \\
\hline
\end{tabular}

Table 1: The association between the two discursive categories and the three perspectives

Relative Norm Distance (RND) metric was applied to check the association between the neutral word lists and the two discursive categories. Positive values indicate stronger association with the "immigrant" category, and negative values with the "refugee" category. 


\begin{tabular}{|c|c|}
\hline Cluster & Keywords \\
\hline $\begin{array}{l}\text { 1. Crime and illegality } \\
(78193)\end{array}$ & $\begin{array}{l}\text { Sweden, the police, year(-s), persons, Migration agency, ac- } \\
\text { cording to, child(-ren), Afghanistan, Swedish, crime, the man, } \\
\text { unaccompanied, expulsion, earlier, asylum seeker(-s), prison, } \\
\text { Malmö, men, residence permit(-s), expelled, ISIS, case(-s), the } \\
\text { country, SVT, despite, police, people, woman, Stockholm, year } \\
\text { old }\end{array}$ \\
\hline $\begin{array}{l}\text { 2. Finances and welfare } \\
(105732)\end{array}$ & $\begin{array}{l}\text { Sweden, year(-s), EU, the government, Swedish, newcomers, } \\
\text { people, SD, needs, child(-ren), job(-s), new, writes, whole, pro- } \\
\text { posal, billions, municipalities, good, millions, see, Sweden's, } \\
\text { crowns, big, money, percent, refugees, better, according to, } \\
\text { sees, politics }\end{array}$ \\
\hline $\begin{array}{l}\text { 3. Civil activity and non- } \\
\text { profit sector (133862) }\end{array}$ & $\begin{array}{l}\text { Welcome, see, welcome, year(-s), Saturday, Sweden, Roma, } \\
\text { Swedish, Stockholm, new, place, miss, day, Malmö, tonight, } \\
\text { read, music, diversity, Sunday, Arabic, whole, warmly, more- } \\
\text { over, Friday, children, seen, o'clock, Thursday, evening, year's }\end{array}$ \\
\hline $\begin{array}{l}\text { 4. Migrant reception } \\
\text { and immigration policy } \\
(242249)\end{array}$ & $\begin{array}{l}\text { Sweden, writes, Swedish, SD, read, year(-s), good, see, new, } \\
\text { refugees, EU, unaccompanied, child(-ren), people, today's, } \\
\text { Sweden's, Afghanistan, day, new, Syria, whole, sees, SVT, } \\
\text { newcomers, share, the Migration Agency, stands, asylum } \\
\text { seeker(-s), immigrant(-s), Swedish }\end{array}$ \\
\hline $\begin{array}{l}\text { 5. Integration of newcom- } \\
\text { ers (104691) }\end{array}$ & $\begin{array}{l}\text { Newcomers, Swedish, Sweden, child(-ren), integration, new, } \\
\text { read, looks for, year(-s), work, diversity, municipality, good, } \\
\text { students, SFI, see, people, unaccompanied, needs, help, young, } \\
\text { job(-s), young people, questions, moreover, works, teacher(-s), } \\
\text { information, learn, project }\end{array}$ \\
\hline 6. Help refugees (107569) & $\begin{array}{l}\text { Child(-ren), Sweden, year(-s), people, to help, help, needs, } \\
\text { good, little, Syria, life(-ves), day, Allah, see, Swedish, } \\
\text { home(-s), refugees, give, whole, money, family, looks, food, } \\
\text { Afghanistan, unaccompanied, escape, the children, lives, coun- } \\
\text { try, clothes }\end{array}$ \\
\hline 7. Global politics (85367) & $\begin{array}{l}\text { Syria, Sweden, USA, EU, Israel, Afghanistan, year(-s), ISIS, } \\
\text { people, Europe, countries, Iraq, refugees, Swedish, UN, Turkey, } \\
\text { the country, world, according to, al, child(-ren), war(-s), coun- } \\
\text { try, Russia, millions, whole, Christian, Muslim, the govern- } \\
\text { ment, Jews }\end{array}$ \\
\hline $\begin{array}{l}\text { 8. Racism and multicul- } \\
\text { turalism (125698) }\end{array}$ & $\begin{array}{l}\text { Sweden, SD, Swedish, people, country, Islam, immigrant(-s), } \\
\text { people, sees, Muslims, politicians, year(-s), party, see, whole, } \\
\text { women, child(-ren), Swedish, good, Swedes, stands, racism, the } \\
\text { society, should, writes, politics, the people, wrong, the country, } \\
\text { Löfven }\end{array}$ \\
\hline
\end{tabular}

Table 2: Thematic structure of the Facebook discourse (a solution with eight clusters)

Document clusters were identified with the help of K-means clustering, and keywords with the help of TF-IDF weighting. 
some of the messages do construct migrant identities in a seemingly positive light, as it can be exemplified by the following piece, although the distinction between immigrants and the Swedish is in most cases preserved:

(2) We should not forget that we have many good immigrants better than many Swedes.

When it comes to the refugee category, this group's right to reside in Sweden is not questioned in the same ways as the immigrants', although, in this case, a certain boundary is created between the "real" refugees who qualify for protection and help versus immigrants who claim to be refugees and are thus not entailed to the same rights as the first group:

(3) Migrants pretended to be Syrian refugees, stole asylum places from the real refugees.

Another observation concerning the discursive construction of the refugee category in the corpus is that Facebook users tend to represent refugee reception and management of refugee flows either as a matter of international or national concern that needs to be addressed or as an object of administrative interventions. Since the dataset spans the messages that were published during the 2014/2016 refugee crisis, references to statistics and forecasts on the number of arrivals, as well as news on other countries' refugee reception policies, seem to represent quite typical ways for the contextualisation of the refugeehood, as follows from the following piece:

(4) Eksjö municipality will increase the number of asylum places for unaccompanied children next year.

\subsection{Interpretative repertoires in the Facebook discourse}

Another way to look at the corpus is to identify its interpretative repertoires. The use of computational methods helps automate this process without reading large pieces of text, which is especially relevant in social media studies. At the same time, the use of doc2vec allows to "zoom in" the content of the corpus by choosing a higher number of clusters and by reading some of the most relevant pieces closely.

To achieve this goal, the documents were divided into eight clusters, as explained in the Methods section. The keywords for each of the document clusters were obtained with the help of TF-IDF weighting and are presented in Table 2. Generally speaking, the main repertoire themes can be divided into several groups: those discussing international relations and conflicts (cluster 7 ), those in one way or another discussing the reception and integration of the newcomers in Sweden (clusters 2, 4, 5, 8), and a group of documents that are specifically focused on the assistance and help to the refugees (clusters 3 and 6). On top of this, one can also distinguish a smaller group of documents that discuss migration in the context of potential threat to the Swedish society (cluster 1, and, partly, cluster 8). 
The issues of integration and reception of migrants in Sweden seem to have one of the central roles in the discourse, and it also has the biggest size with respect to the number of documents since around two-thirds of all documents discuss it in one way or the other. The following pieces exemplify typical utterances:

(5) Will we get a proposal to create an integration school from the politicians in this election round? (Cluster 4 - Migrant reception and immigration policy)

(6) How do you work with the intercultural approach and how does it look in your environment? (Cluster 5 - Integration of newcomers)

In contrast to the major part of the documents that represent immigration as a matter of primarily Sweden's national concern, another group of messages is also focused on the conflicts and crises elsewhere in the world. Yet, as exemplified by the utterance below, this topic is mainly contextualised as a matter of international politics and neglects the experiences of people directly affected by the instability and warfare:

(7) EU and Nato support the attacks. Syria and Russia condemn them. That's what has happened (Cluster 7 - Global politics)

A sufficiently large part of the posts centres around the assistance to the refugees both in Sweden and abroad, which is not surprising given the media resonance that the refugee crisis caused in the European countries in 2014-2016 and the wave of public support for the refugees that was more typical for the first part of the crisis (i.e. until the end of 2015). Social media in general and Facebook in particular could be quite naturally seen as the most popular and effective platforms to coordinate the effort of individual activists and the non-profit sector:

(8) Hi, I have some clothes and shoes that I would like to donate to refugees. But I don't really know where I can bring them. So I wonder if there's anybody who knows? (Cluster 6 - Help refugees)

(9) Welcome to a multicultural party on Saturday in Västerås! (Cluster 2 - Civil activity and non-profit sector)

Finally, a minor part of the messages in the corpus discusses migration in the context of potential threat to the Swedish society, primarily concerning illegal entry into the country, deportations and commitment of crimes:

(10) THE PROSECUTOR REQUESTED DEPORTATION THE MAN FULLY FREED (Cluster 1 - Crime and illegality)

This, however, does not mean that the rest of the messages in the corpus take a positive stance on the topic - in fact, the assignment of documents to clusters happens irrespective of the messages' general stances or sentiments, as demonstrated 
by the utterance below:

(11) DON'T WANT TO HAVE MORE ASYLUM SEEKERS AND IMMIGRANTS IN SWEDEN IT IS ENOUGH (Cluster 4 - Migrant reception and immigration policy)

In this way, immigration discourse cannot be described as fully coherent. Rather, it consists of several possible interpretations of the topic that can clash or contradict each other.

\section{Discussion}

Summing up the results of the analysis outlined in the previous section, it seems that the interpretative repertoire used to contextualise the immigration topic on Facebook has many commonalities with the repertoires of other social media platforms and resources, such as Twitter and Flashback forum [51]. Indeed, some of the themes, such as financial aspects, racism or migrant illegality, are echoed by the social media users across these media resources and seem to act as general ways to frame and contextualise the immigration agenda in Sweden. Yet, what makes the Facebook discourse stand out compared to other participatory media is its orientation towards the expression of solidarity and assistance to the newcomers, with two particular interpretations of the topic belonging to this category.

Nevertheless, the study finds that the reproduction of migrant otherness is a distinctive trait of the Swedish Facebook discourse, yet, there seems to be a large gap between the ways in which the images of immigrants and refugees are created in the corpus. This conclusion supports the results of previous work that finds that migrantness and refugeehood represent two distinct social categories that imply different consequences and status positions for their holders [12, 13]. At the same time, the presence of these competitive narratives of solidarity with migrants versus the narratives of migrant otherness provides some evidence for a possible polarisation of the Facebook audience. This suggestion can be supported by results of previous studies finding that social media audiences are considerably polarised based on their ideological stances [52].

Although some of the existing studies highlight the difference between the deserving refugees and undeserving migrants [11, I find that the idea of deservingness is more typical for the immigrant label. The concepts of criminality or threat, as well as costs and benefits, seem to be typically activated in the construction of deserving versus undeserving immigrants. Criminal record, type of entry, level of education and ability to pay taxes are the factors that seem to play the key role in this bordering process that, according to the Facebook users, defines whether particular groups of immigrants are to be tolerated. Open racism, in this case, is covered and rationalised by the argumentative character of the messages and extensive appeals to numbers and statistics, as well as references to external resources, which is argued to be one of the most basic discursive strategies [2, p. 35].

On the other hand, the concept of refugeehood is closely related to the idea that this is a matter that needs to be managed and administered, as demonstrated in the previous section. At the same time, solidaristic framing of the refugee reception is still quite common in the corpus. Yet, as argued by the previous research, the discourses 
on refugeehood mostly ignore the voices and lived experiences of those categorised as refugees [53. The topic of refugee reception, then, is turned into a matter of Sweden's national concern that needs to be addressed or as a subject of policy implementation, rather as an issue for those assigned with these categories. This argument can be supported by the results of previous studies that find "institutional framing" of the refugee agenda in Sweden [54].

Accordingly, what unites the representation of both of these discursive categories is their objectification. As pointed out by the earlier research, objectification can be called a typical strategy for the construction of images of the Other [55], although, in this case, it seems to work in different ways for those identified as immigrants or refugees. While immigrants are seen as potential sources to contribute to society and to be capitalised upon, refugees, as mentioned above, are constructed as an object of administrative management and policy-making. In this way, those classified as belonging to either of the two categories are in some sense deprived of their agency.

These observations seem to resonate with the approach that distinguishes exploitative and exclusionary modes of racism [56]. While the former presumes that only those who can immediately provide net profit to the Swedish society are to be tolerated, the latter suggests that the rest is to be excluded from the society if the immediate creation of net value is not viable or if the costs out-weight potential profit to the society. Researchers refer to this phenomenon as a form of discursive discrimination [55].

While such a form of discursive discrimination is justified with the help of argumentation and reasoning, its ground can be related to the general socio-political context surrounding migrant reception and integration policies in Sweden. As noted in the introductory section, the competition between the narratives of old Sweden and good Sweden [26] seems to play out in this particular case of the immigration discourse and, to some extent, can be described as users' diverging stances on the topic. Further, as argued by the social researchers, the discourses on immigration in the Nordic countries are centred around its potential challenges to the welfare state [30] that has lately undergone sufficient changes in Sweden in favour of more neo-liberal social policy [57]. This observation can be coupled with a statement about a prevailing sense of failure of multiculturalism and shrinking public support for the multiculturalist project [58]. Thus, the Facebook discourse seems to reflect such processes that take place in Swedish society.

Nevertheless, as argued above, social media represent contradictory public arenas, and the results of the analysis quite evidently demonstrate these contradictions. As pointed out before, social networking sites can be used to de-monopolise and acquire discursive power in order to challenge common preconceptions about the Other [37], which, however, does not seem to be the case for the Swedish Facebook discourse. On the contrary, one can argue that discursive power on Facebook is exercised to sustain existing assumptions about the discursive categories in question, as demonstrated in the previous section. On the other hand, the researchers propose that new media facilitate the development of participatory culture [59], and, in Sweden, the use of social media during the refugee crisis to support grass-roots activism has been demonstrated before [60]. This work admittedly finds that the Facebook platform has enabled the expression of solidarity with refugees.

On the other hand, a particular trait of the technological design of new media is 
their tendency to the fragmentation of audiences (and, possibly, discourses) and the emergence of echo-chambers that provide favourable conditions for users' radicalisation [61, 62], or, as a separate case, the polarisation of the audiences [52]. Indeed, I conclude that the Facebook discourse represents a set of competing contextualisations of the topic and find a distinct set of explicitly or implicitly biased messages in the corpus, which, on the other hand, goes in line with the assumptions of the discourse theory about the presence of competing narratives as part of any interpretative repertoire [34, p. 219]. Nevertheless, this evidence allows hypothesising the fragmentation of the Facebook audience. Digital racism and the prevention of potential spill-over of this form of racism into the mainstream discourse thus represent the challenges that need yet to be addressed in social media studies.

\section{Conclusion}

Summing up the findings of this paper, it seems that Facebook plays a contradictory role in the construction and reproduction of migrant categories. On the one hand, Facebook seems to enable the formation of affective publics and participatory culture. On the other hand, it is still active in the re-enactment of migrant otherness as well as biased and racialised forms of migrants' representations that can be characterised as forms of discursive violence.

From the methodological point of view, this paper's ambition has been to demonstrate the possibilities for the use of vector space models in social media and discourse studies. On the one hand, word embeddings worked as a powerful tool that helped to get a glance at the existing repertoires, as well as to conceive of meanings of individual labels. On top of this, it provided an opportunity to combine distant reading of the whole corpus with close reading of individual documents, at the same time eliminating researcher bias with regard to the choice of particular documents.

Since the application of word embeddings in media and discourse studies is still much a work in progress, this opens up possibilities for developing new frameworks for the analysis of social media discourses with the help of this method. This can be seen as an exciting possibility for future research and suggests a path for a successful combination of corpus linguistics with discourse studies. 


\section{References}

[1] T. A. Van Dijk, "Mediating racism: The role of the media in the reproduction of racism," Language, Power and Ideology, vol. 7, 1989.

[2] T. A. Van Dijk, "Stories and racism," Narrative and Social Sontrol: Critical Perspectives, vol. 21, pp. 121-142, 1993.

[3] M. Wahlström and A. Törnberg, "Social media mechanisms for right-wing political violence in the 21st century: Discursive opportunities, group dynamics, and coordination," Terrorism and Political Violence, pp. 1-22, 2019.

[4] J. R. Firth, "A synopsis of linguistic theory 1930-1955, volume 1952-59," The Philological Society, 1957.

[5] N. Garg, L. Schiebinger, D. Jurafsky, and J. Zou, "Word embeddings quantify 100 years of gender and ethnic stereotypes," in Proceedings of the National Academy of Sciences of the United States of America, vol. 115.

[6] L. Rheault and C. Cochrane, "Word embeddings for the analysis of ideological placement in parliamentary corpora," Political Analysis, vol. 28, no. 1, p. 112-133, 2020 .

[7] I. Brigadir, D. Greene, and P. Cunningham, "Analyzing discourse communities with distributional semantic models," in Proceedings of the ACM Web Science Conference, (New York, NY, USA), Association for Computing Machinery, 2015.

[8] L. Viola and J. Verheul, "One hundred years of migration discourse in the Times: A discourse-historical word vector space approach to the construction of meaning," Frontiers in Artificial Intelligence, vol. 3, p. 64, 2020.

[9] T. Hübinette and C. Lundström, "Three phases of hegemonic whiteness: Understanding racial temporalities in Sweden," Social Identities, vol. 20, no. 6, pp. 423437, 2014.

[10] K. Borevi, "The political dynamics of multiculturalism in Sweden," in Challenging Multiculturalism: European Models of Diversity (R. Taras, ed.), pp. 138-162, Edinburgh University Press, 2013.

[11] S. M. Holmes and H. Castañeda, "Representing the 'European refugee crisis' in Germany and beyond: Deservingness and difference, life and death," American Ethnologist, vol. 43, no. 1, pp. 12-24, 2016.

[12] S. Goodman, A. Sirriyeh, and S. McMahon, "The evolving (re)categorisations of refugees throughout the 'refugee/migrant crisis'," Journal of Community \& Applied Social Psychology, vol. 27, no. 2, pp. 105-114, 2017.

[13] C. Kyriakides, "Words don't come easy: Al Jazeera's migrant-refugee distinction and the European culture of (mis)trust," Current Sociology, vol. 65, no. 7, pp. 933$952,2017$. 
[14] R. Kreis, "\#refugeesnotwelcome: Anti-refugee discourse on Twitter," Discourse $\&$ Communication, vol. 11, no. 5, pp. 498-514, 2017.

[15] M. G. Galantino, "The migration-terrorism nexus: An analysis of German and Italian press coverage of the 'refugee crisis'," European Journal of Criminology, 2020 .

[16] A. Findor, M. Hruška, P. Jankovská, and M. Pobudová, "Re-examining public opinion preferences for migrant categorizations: "refugees" are evaluated more negatively than "migrants" and "foreigners" related to participants' direct, extended, and mass-mediated intergroup contact experiences," International Journal of Intercultural Relations, vol. 80, pp. 262-273, 2021.

[17] D. D. Coninck, "Migrant categorizations and European public opinion: Diverging attitudes towards immigrants and refugees," Journal of Ethnic and Migration Studies, vol. 46, no. 9, pp. 1667-1686, 2020.

[18] H. Crawley and D. Skleparis, "Refugees, migrants, neither, both: Categorical fetishism and the politics of bounding in Europe's 'migration crisis'," Journal of Ethnic and Migration Studies, vol. 44, no. 1, pp. 48-64, 2018.

[19] C. Lundström, "The white side of migration: Reflections on race, citizenship and belonging in Sweden," Nordic Journal of Migration Research, vol. 7, no. 2, pp. 79 87, 2017.

[20] G. Myrberg, "Who is an immigrant?," in Diversity, Inclusion and Citizenship in Scandinavia (B. Bengtsson, P. Strömblad, and A.-H. Bay, eds.), pp. 47-74, Cambridge Scholars Publisher, 2010.

[21] S. Merrill and M. Åkerlund, "Standing up for Sweden? The racist discourses, architectures and affordances of an anti-immigration Facebook group," Journal of Computer-Mediated Communication, vol. 23, no. 6, pp. 332-353, 2018.

[22] F. R. Ammaturo, "Europe and whiteness: Challenges to European identity and European citizenship in light of Brexit and the 'refugees/migrants crisis'," European Journal of Social Theory, vol. 22, no. 4, pp. 548-566, 2019.

[23] G. Delanty, R. Wodak, and P. Jones, Identity, Belonging and Migration. Liverpool University Press, 2008.

[24] N. D. Genova, "The 'migrant crisis' as racial crisis: Do Black Lives Matter in Europe?," Ethnic and Racial Studies, vol. 41, no. 10, pp. 1765-1782, 2018.

[25] N. De Genova, M. Tazzioli, and S. Álvarez-Velasco, "Europe/crisis: New keywords of 'the crisis' in and of 'Europe'," Near Futures Online, vol. 1, pp. 11-45, 2016.

[26] T. Hübinette and C. Lundström, "Sweden after the recent election: The doublebinding power of Swedish whiteness through the mourning of the loss of 'old Sweden' and the passing of 'good Sweden'," NORA - Nordic Journal of Feminist and Gender Research, vol. 19, no. 1, pp. 42-52, 2011. 
[27] Y. Erdogan-Ozturk and H. Isik-Guler, "Discourses of exclusion on Twitter in the Turkish context: \#ülkemdesuriyeliistemiyorum (\#idontwantsyriansinmycountry)," Discourse, Context \& Media, vol. 36, 2020.

[28] M. Ekman, "Anti-immigration and racist discourse in social media," European Journal of Communication, vol. 34, no. 6, pp. 606-618, 2019.

[29] A. Törnberg and M. Wahlström, "Exploring framing and identity in an online antiimmigrant discussion group," Sociologisk Forskning, vol. 55, no. 2-3, pp. 267-292, 2018.

[30] I. Sakki and K. Pettersson, "Discursive constructions of otherness in populist radical right political blogs," European Journal of Social Psychology, vol. 46, no. 2, pp. 156-170, 2016.

[31] J. Potter and M. Wetherell, Discourse and Social Psychology: Beyond Attitudes and Behaviour. SAGE, 1987.

[32] M. Wetherell, Mapping the Language of Racism: Discourse and the Legitimation of Exploitation. Columbia University Press, 1992.

[33] N. Edley and M. Wetherell, "Jekyll and Hyde: Men's constructions of feminism and feminists," Feminism \& Psychology, vol. 11, no. 4, pp. 439-457, 2001.

[34] K. Boréus, Analyzing Text and Discourse: Eight Approaches for the Social Sciences. SAGE, 2017.

[35] D. Tannen, H. E. Hamilton, and D. Schiffrin, The Handbook of Discourse Analysis. Wiley Blackwell, 2015.

[36] S. Jänicke, G. Franzini, M. F. Cheema, and G. Scheuermann, "On close and distant reading in digital humanities: A survey and future challenges," in Eurographics Conference on Visualization (EuroVis) - STARs (R. Borgo, F. Ganovelli, and I. Viola, eds.), pp. 83-103, The Eurographics Association, 2015.

[37] M. KhosraviNik and E. Esposito, "Online hate, digital discourse and critique: Exploring digitally-mediated discursive practices of gender-based hostility," Lodz Papers in Pragmatics, vol. 14, no. 1, pp. 45-68, 2018.

[38] Z. S. Harris, "Distributional structure," Word, vol. 10, no. 2-3, pp. 146-162, 1954.

[39] C. Fan, "Crowdtangle for academics and researchers," Facebook, 2021. https://help.crowdtangle.com/en/articles/4302208-crowdtangle-for-academicsand-researchers.

[40] A. Joulin, E. Grave, P. Bojanowski, and T. Mikolov, "Bag of tricks for efficient text classification," arXiv.org, 2016. https://arxiv.org/abs/1607.01759.

[41] T. Mikolov, K. Chen, G. Corrado, and J. Dean, "Efficient estimation of word representations in vector space," arXiv.org, 2013. https://arxiv.org/abs/1301.3781. 
[42] Q. V. Le and T. Mikolov, "Distributed representations of sentences and documents," arXiv.org, 2014. https://arxiv.org/abs/1405.4053.

[43] R. Řehưřek and P. Sojka, "Software framework for topic modelling with large corpora," in Proceedings of the LREC 2010 Workshop on New Challenges for NLP Frameworks, pp. 45-50, ELRA, 2010.

[44] Y. Tsvetkov, M. Faruqui, and C. Dyer, "Correlation-based intrinsic evaluation of word vector representations," in Proceedings of the 1st Workshop on Evaluating Vector-Space Representations for NLP, pp. 111-115, Association for Computational Linguistics, 2016.

[45] P. Fallgren, J. Segeblad, and M. Kuhlmann, "Towards a standard dataset of Swedish word vectors," in Sixth Swedish Language Technology Conference (SLTC), 2016.

[46] L. Borin and M. Forsberg, "All in the family: A comparison of SALDO and WordNet," in Proceedings of the Nodalida 2009 Workshop on WordNets and other Lexical Semantic Resources - between Lexical Semantics, Lexicography, Terminology and Formal Ontologies, vol. 7 of NEALT Proceedings Series, 2009.

[47] E. Altszyler, S. Ribeiro, M. Sigman, and D. Fernández Slezak, "The interpretation of dream meaning: Resolving ambiguity using latent semantic analysis in a small corpus of text," Consciousness and Cognition, vol. 56, pp. 178 - 187, 2017.

[48] E. Dusserre and M. Padró, "Bigger does not mean better! We prefer specificity," in 12th International Conference on Computational Semantics (IWCS), 2017.

[49] O. Levy, Y. Goldberg, and I. Dagan, "Improving distributional similarity with lessons learned from word embeddings," Transactions of the Association for Computational Linguistics, vol. 3, pp. 211-225, 2015.

[50] P. Badilla, F. Bravo-Marquez, and J. Pérez, "Wefe: The word embeddings fairness evaluation framework," in Proceedings of the Twenty-Ninth International Joint Conference on Artificial Intelligence, IJCAI-20, pp. 430-436, International Joint Conferences on Artificial Intelligence Organization, 2020.

[51] V. Yantseva, "Migration discourse in Sweden: Frames and sentiments in mainstream and social media," Social Media + Society, vol. 6, no. 4, 2020.

[52] J. Kaiser and C. Puschmann, "Alliance of antagonism: Counterpublics and polarization in online climate change communication," Communication and the Public, vol. 2, no. 4, pp. 371-387, 2017.

[53] L. Chouliaraki and R. Zaborowski, "Voice and community in the 2015 refugee crisis: A content analysis of news coverage in eight European countries," International Communication Gazette, vol. 79, no. 6-7, pp. 613-635, 2017.

[54] D. Abdelhady, "Media constructions of the refugee crisis in Sweden: Institutions and the challenges of refugee governance," in Refugees and the Violence of Welfare Bureaucracies in Northern Europe (D. Abdelhady, N. Gren, and M. Joormann, eds.), Manchester University Press, 2020. 
[55] K. Boréus, "Nationalism and discursive discrimination against immigrants in Austria, Denmark and Sweden," in Right-Wing Populism in Europe: Politics and Discourse (R. Wodak, M. KhosraviNik, and B. Mral, eds.), p. 293-308, Bloomsbury Academic, 2013.

[56] D. Mulinari and A. Neergaard, "Theorising racism: Exploring the Swedish racial regime," Nordic Journal of Migration Research, vol. 7, no. 2, 2017.

[57] C.-U. Schierup and A. Ålund, "The end of Swedish exceptionalism? Citizenship, neoliberalism and the politics of exclusion," Race \& Class, vol. 53, no. 1, pp. 45-64, 2011.

[58] M. Dahlstedt and A. Neergaard, "Crisis of solidarity? Changing welfare and migration regimes in Sweden," Critical Sociology, vol. 45, no. 1, pp. 121-135, 2019.

[59] Y. Benkler, The Wealth of Networks: How Social Production Transforms Markets and Freedom. Yale University Press, 2006.

[60] A. Kaun and J. Uldam, "Volunteering is like any other business': Civic participation and social media," New Media ES Society, vol. 20, no. 6, pp. 2186-2207, 2018.

[61] D. Batorski and I. Grzywińska, "Three dimensions of the public sphere on Facebook," Information, Communication 6 Society, vol. 21, no. 3, pp. 356-374, 2018.

[62] K. Garimella, G. D. F. Morales, A. Gionis, and M. Mathioudakis, "The ebb and flow of controversial debates on social media," arXiv.org, 2017. https://arxiv.org/abs/1703.05994. 